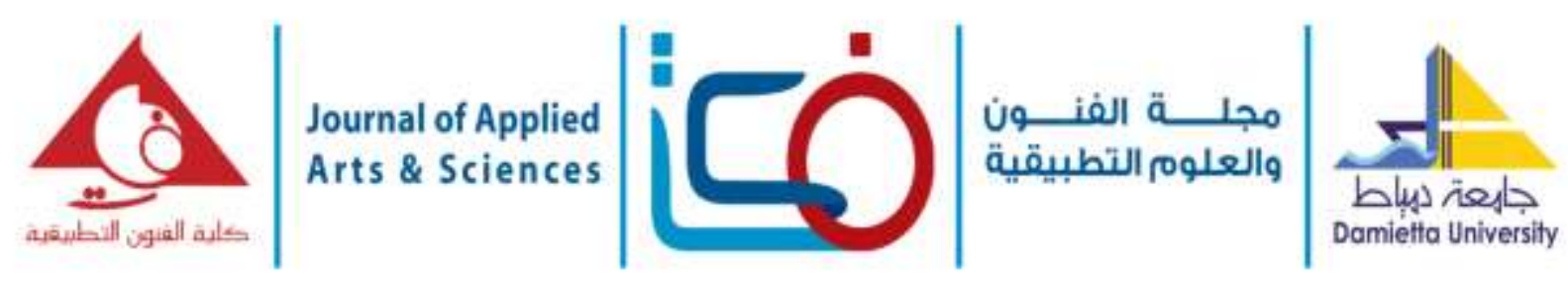

أثر تكنولوجيا الواقع المعزز على التصميم الداخلى

The impact of augmented reality technology on the interior design

\author{
سما رابح عزت محمد رخا \\ معيدة بقسم التصميم الداخلى والأثاث ـ كلية الفنون التطبيقية - جامعة دمياط. \\ ياسر على معبد فرغلى الألى \\ أستاذ نظريات التصميم الداخلى بقسم التصميم الداخلى و الأثاثل ـ كلية الفنون التطبيقية - جامعة دمياط.

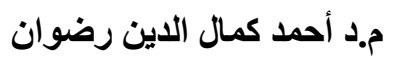 \\ مدرس بقسم التصميم الداخلي والأثاث ـ كلية الفنون التطبيقية - جامعة دمياط.
}

ملفص البعث:

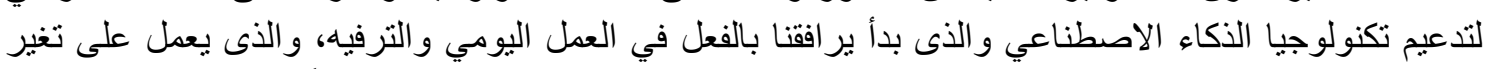

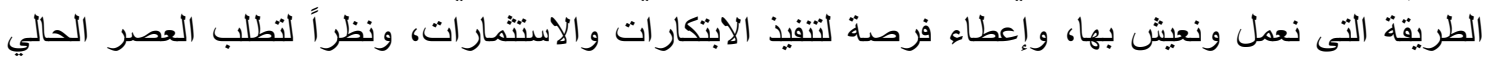

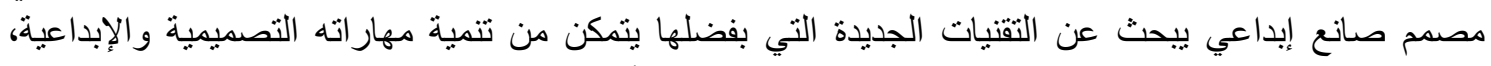
وبإدراج الوسائط الرقمية في العملية التصميمية وتعليم التصميم أدى إلى ظهور مجاعلات جديدة في التصميم مثل التصميم بمساعدة الكمبيوتر.

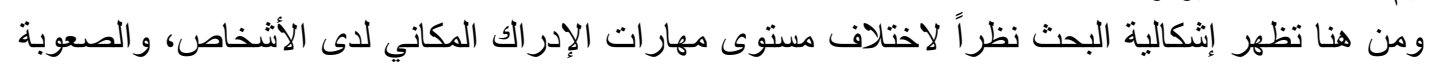

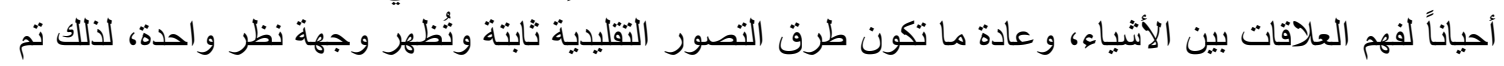

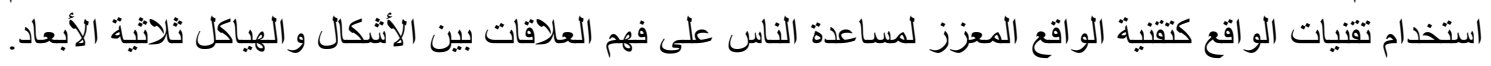

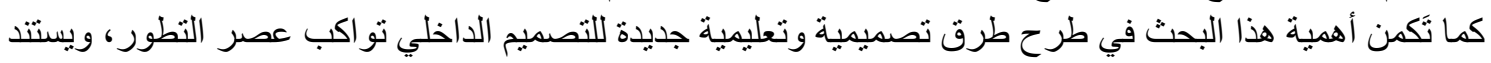

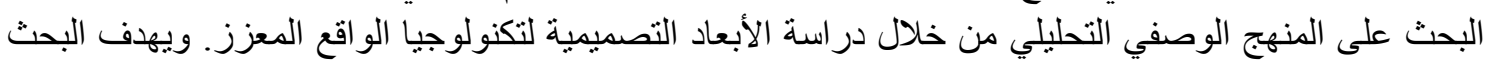

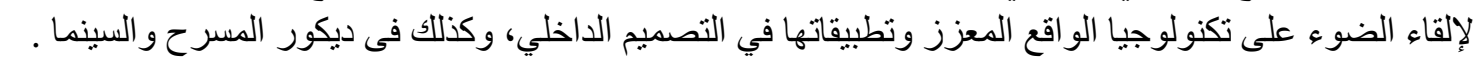

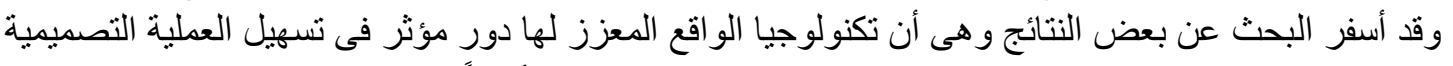

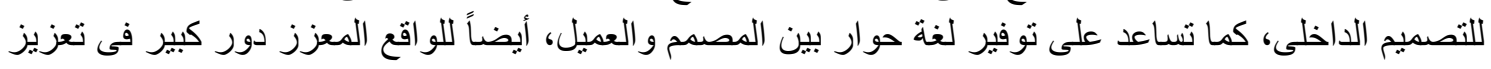

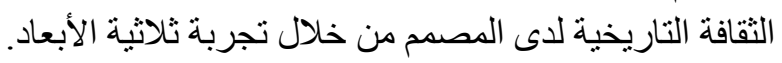
الكلمات المفتاحية: الواقع المعزز ـ ـ تطبيقات الواقع المعزز ـ ـ الواقع الافتراضي ـ ـ التصميم الإفتراضى.

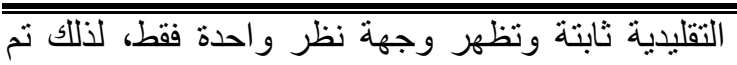

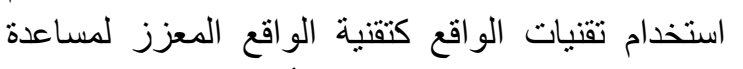
الناس على فهم العلاقات بين الأشكال و الهياكل ثلاثية الونية

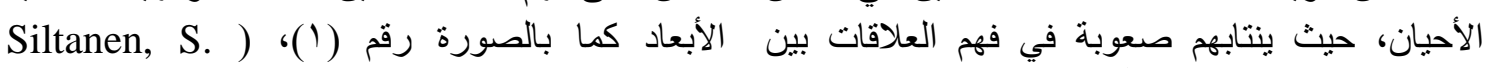
الأشياء المكانية، كما عاداً ما تكون طرق فئن فئ التصور 2015:). 


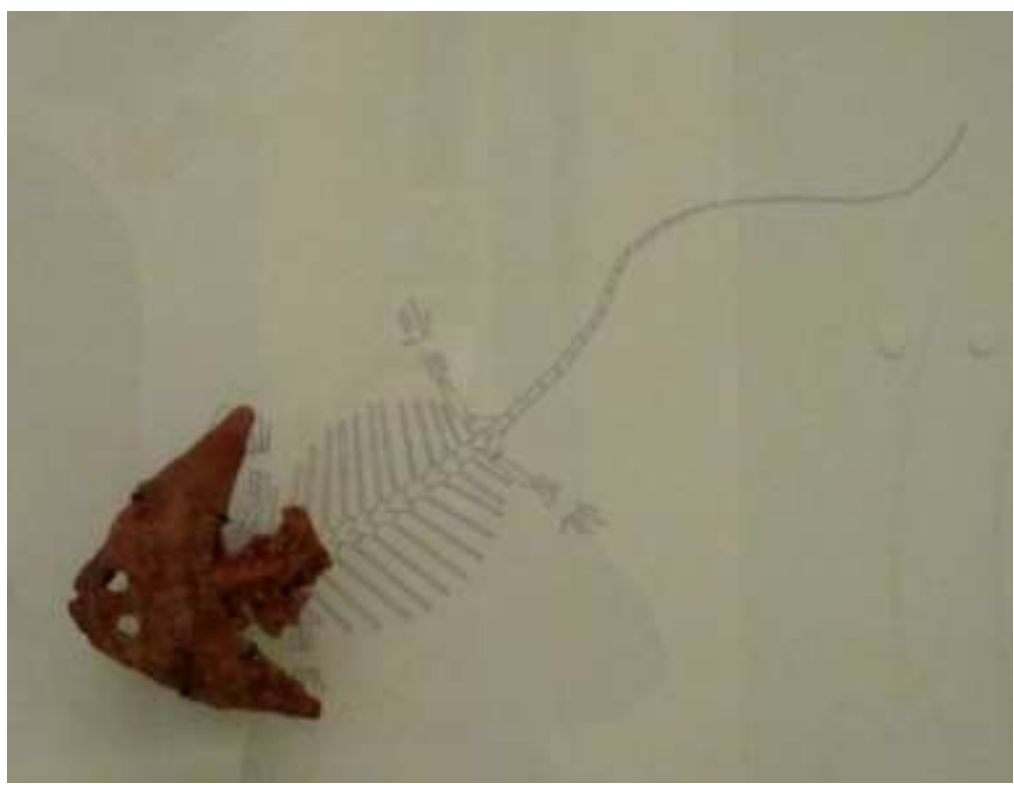

صورة (1) رسم توضيحي لهيكل عظمى لإحدى الحفريات، تساعد المشاهد على فهم

أبعاد الحيوان بصورة أفضل (التصور يوجد في متحف التاريخ الطبيعى، نيويورك)، (Siltanen, S. :2015).

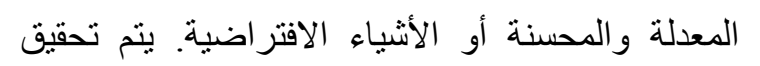
بشكل عام من خلال استخدام الأجهزة المحمولة الألة

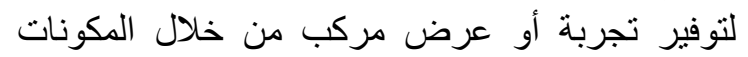

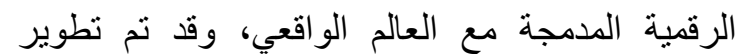

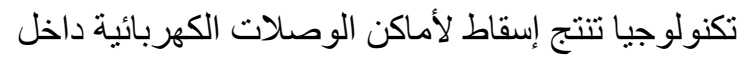

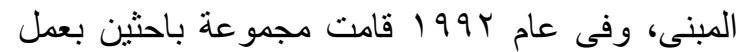

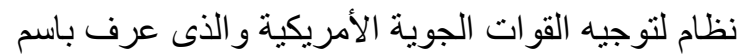
(Virtual Fixtures) على الأسطح وذللك للإستدلال على مواقع الهجوط

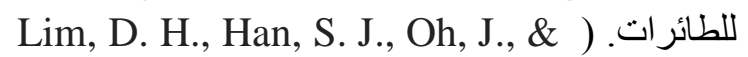

.(Jang, C. S.:2019

مشكلة البحث:

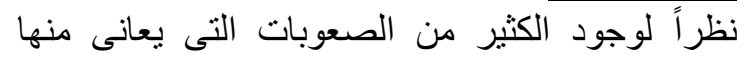
المصمم الداخلي في تحويل فكره إلى واقع ومن ثن الثمان

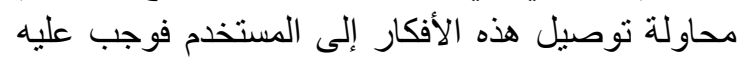

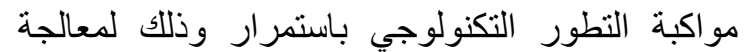

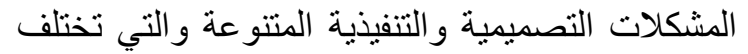
من فراغ لأخر، لذلك تم استخدام تقنيات الو اقع كتقنية التهن

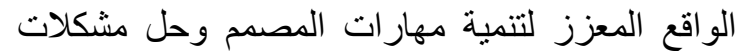

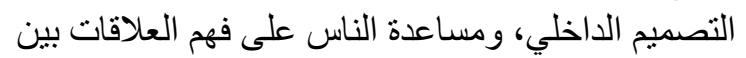
الأشكال و الهياكل ثلاثثية الأبعاد
أدى ظهور تقنية الواقع الافتر اضي فى الستينات على يد إيفان ساذرلاند (Ivan Sutherland) و الذى يعرف

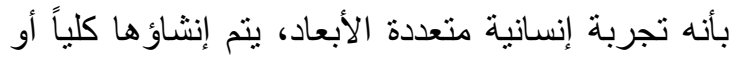

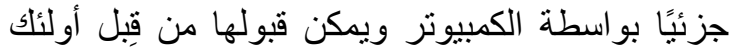

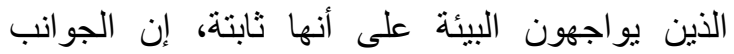

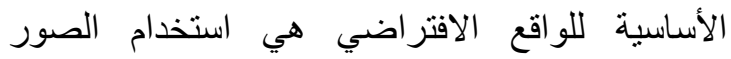
المحاكاة بالحاسوب أو المحاكاة لبناء بيئة إفتراضية، وتحفيز الواجهات الحسية (مثل الصوت، الرؤية). ( Lim, D. H., Han, S. J., Oh, J., \& Jang, C. .(S.:2019 كما أدى تطوير إيفان ساذرلاند (Ivan Sutherland)

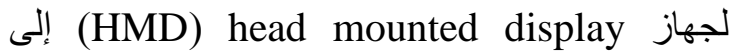
ظهور الو اقع المعزز حيث تَمَكن من إسقاط أثنكال ثلاثية الأبعاد بصورة إظهار سلكى (wireframe) في البيئة

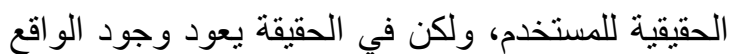
المعزز كفكرة إلى عام 190V حيث ظهر في في أفلام الخيال العلمي وذللك قبل ظهور تقنية الو اقع الافتراضي الوهي

بقوه. ظهر أول مصطلح للواقع المعزز عام •99 19، ويعرف الواقع المعزز Augmented Reality ) بأنه تقنية ناثئة أخرى تدمج الحياة الحقيقية مع الصور بائ 
أهمية البحث: طرح طرق تصميمية وتعليمية جديدة التجارب والأبحاث نحو عملية التصميم المعماري

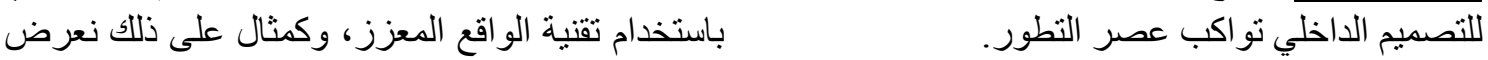

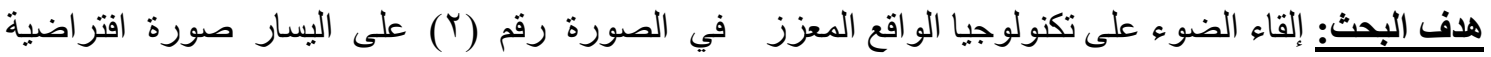
ورصد تطبيقاتها في التصميم الداخلي، وكذلك في ديكور ثلاثية الأبعاد لمنزل بالحجم الحقيقي في بيئة حقيقية،

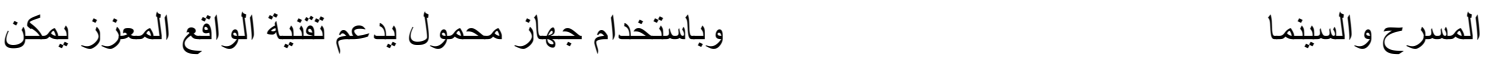

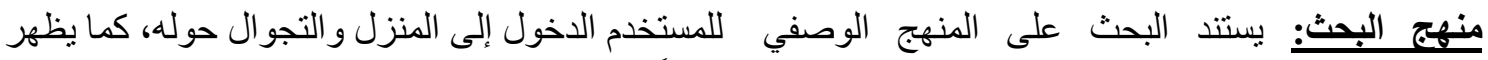

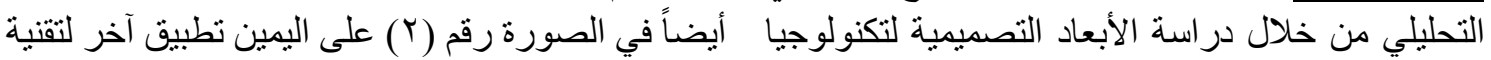

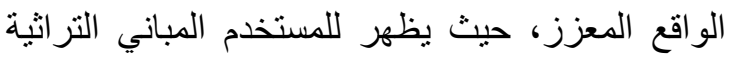
الافتراضية التي تم رفعها بواسطة موقع تاريخي

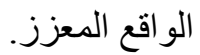

في الآونة الأخيرة ظهرت فيزت تقنية الواقع المعزة كنهج (Phan, V. T., \& Choo, S. Y.: 2010).

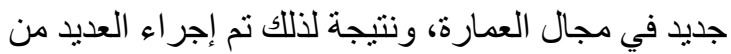

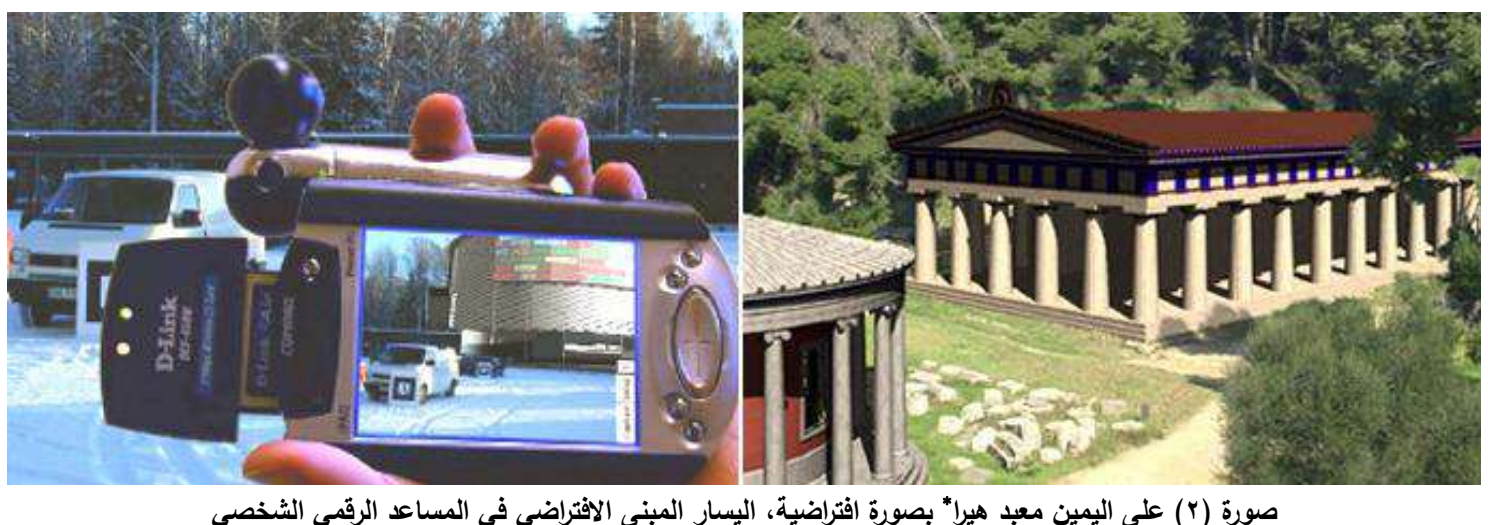

https://www.semanticscholar.org/paper/Interior-Design-in-Augmented-Reality-Environment-Phan )

( Retrieved August,31,2020, 03:50 pm.Choo/6f335173a4608f4e3c38837f8d0c7ed7d0b52208

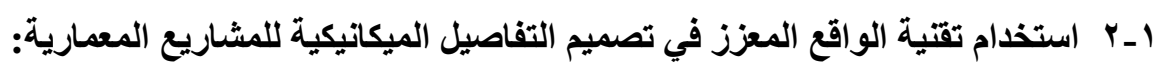

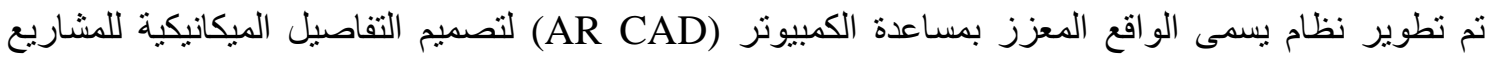

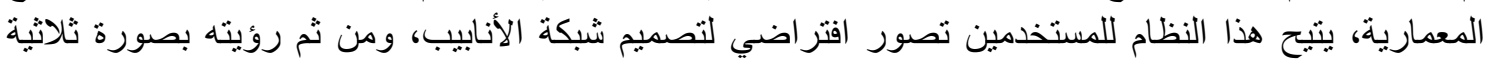

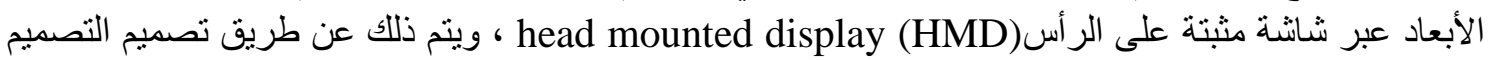

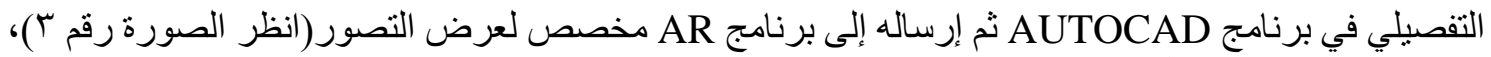

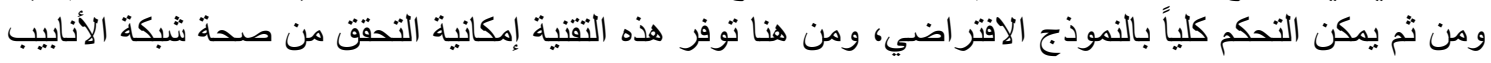
قبل التنفيذ في الو اقع، وبالتالي تسهيل تنفيذ المشاريع المعمارية.(Wang, X.:2009) https://en.wikipedia.org/wiki/Temple of _Hera, Olympia Retrieved August,31,2020, 03:14 pm. 


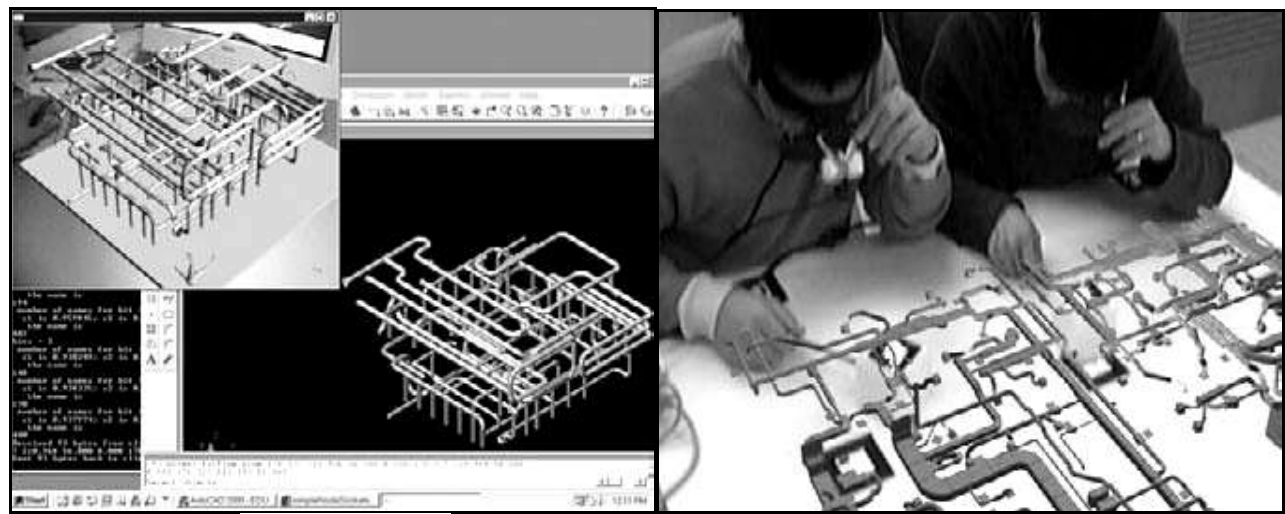

صورة (ץ) تصور إفتراضى في AR CAD ويرنامج أوتوكاد، (Wang, X.2009)..

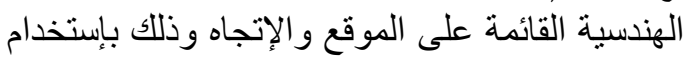

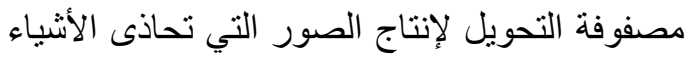

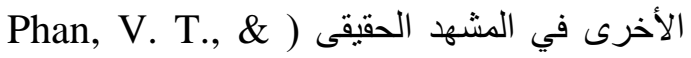
.(2010:Choo, S. Y. r- نماذج تطيقات الواقع المعزز المستخدمة فى التصميم الاخلى:

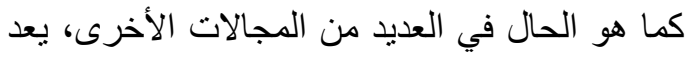

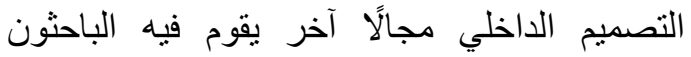

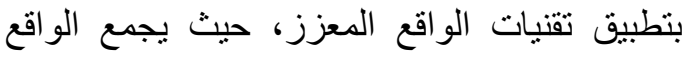

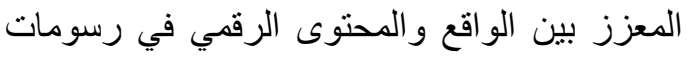

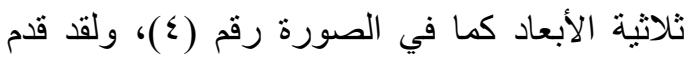

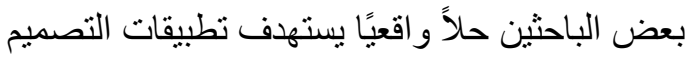

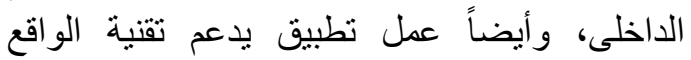

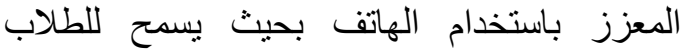
بالتحقق من بعض المباني ومعرفة أنظمتها المختلفة، بالإضافة إلى إمكانية التحقق من جميع المعلومات المنات الخاصة بالمبنى ) Wang, J., Wang, X., Shou,

.(W., Xu, B., Wang, J., \& Xu, B. 2014

r- المعزز: التصيم الداخلى والبيئة الرقمية للواقع يقوم المصدم بشكل أساسي بتطبيق ثلاثة مبادئ

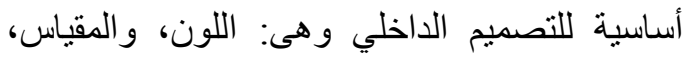

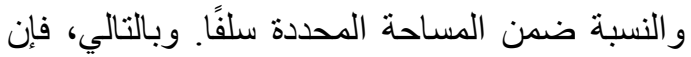

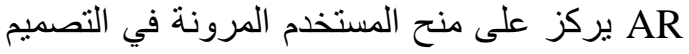
باستخدام هذه المبادئ الأساسية الثناثة، كما يمكن للمستخدم ضبط الخصائص الظاهرية في البيئة المقترحة، وكذلك عناصر الأثاث، وإنشاء ترنيبات Phan, ) مختلفه للأثاث كما لو كان في البيئة الحقيقية وانثاء ترثيات .(2010:V. T., \& Choo, S. Y. يتم بإستخدام تطبيقات CAD إستخر اج المعلومات من الرسم وربطها بقاعدة البيانات، وبالنسبة للمساحة

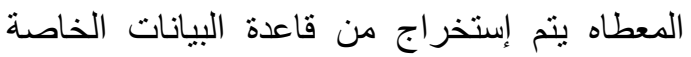

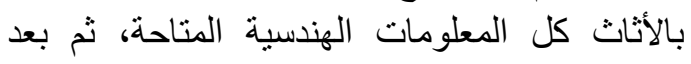
تحميل الأشكال الهندسية يتم حساب مساحة و إتجاه المشهد الخاص بالمستخدم، ثم ينم تحويل البيانات 

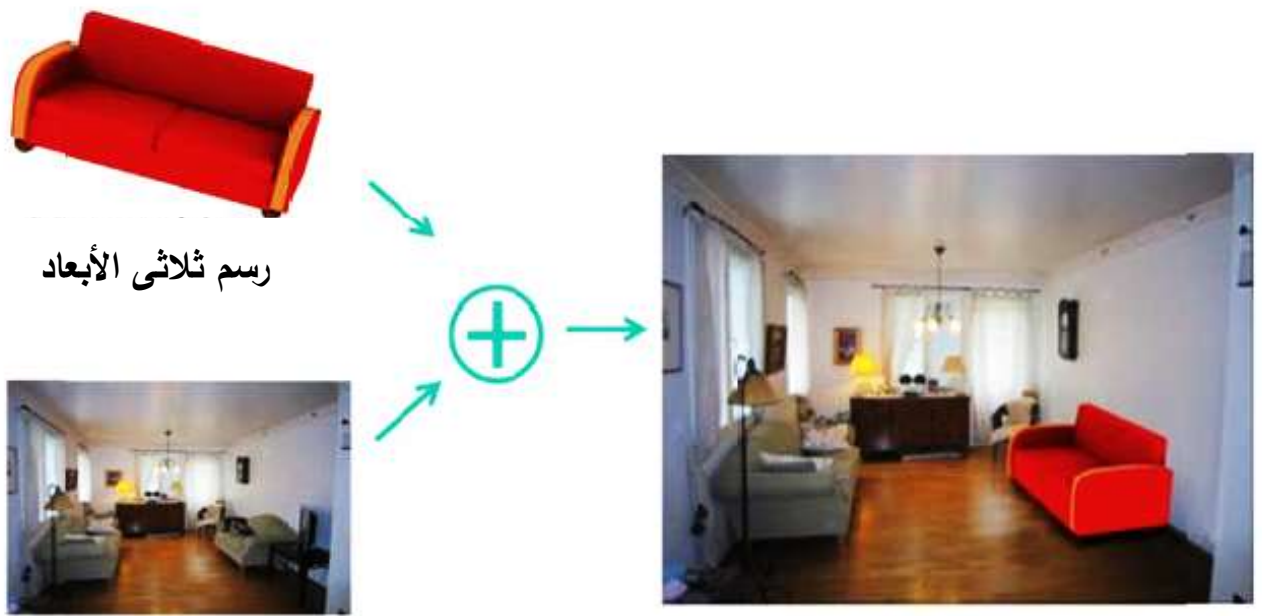

البيئة الحقيقية

\section{الواقع المعزز}

صورة (؛) يجمع الواقع المعزز بين الواقع والمحتوى الرقمي (رسومات ثلاثية الأبعاد) ( 2015.Siltanen, S.).

عملية التصميم الداخلي، بالإضافة إلى تحسين

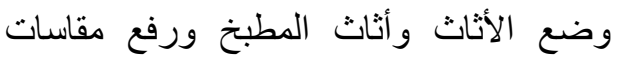

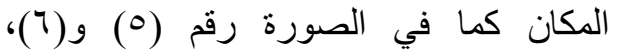

r-ا تطبيق ARKit

شركة Apple مستشعرات عالية الدقة على

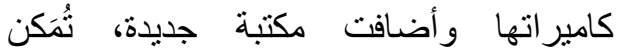

.(Sandu, M., \& Scarlat, I. S. : 2018)

المستخدمين من إستخدام تطبيق ARKit في
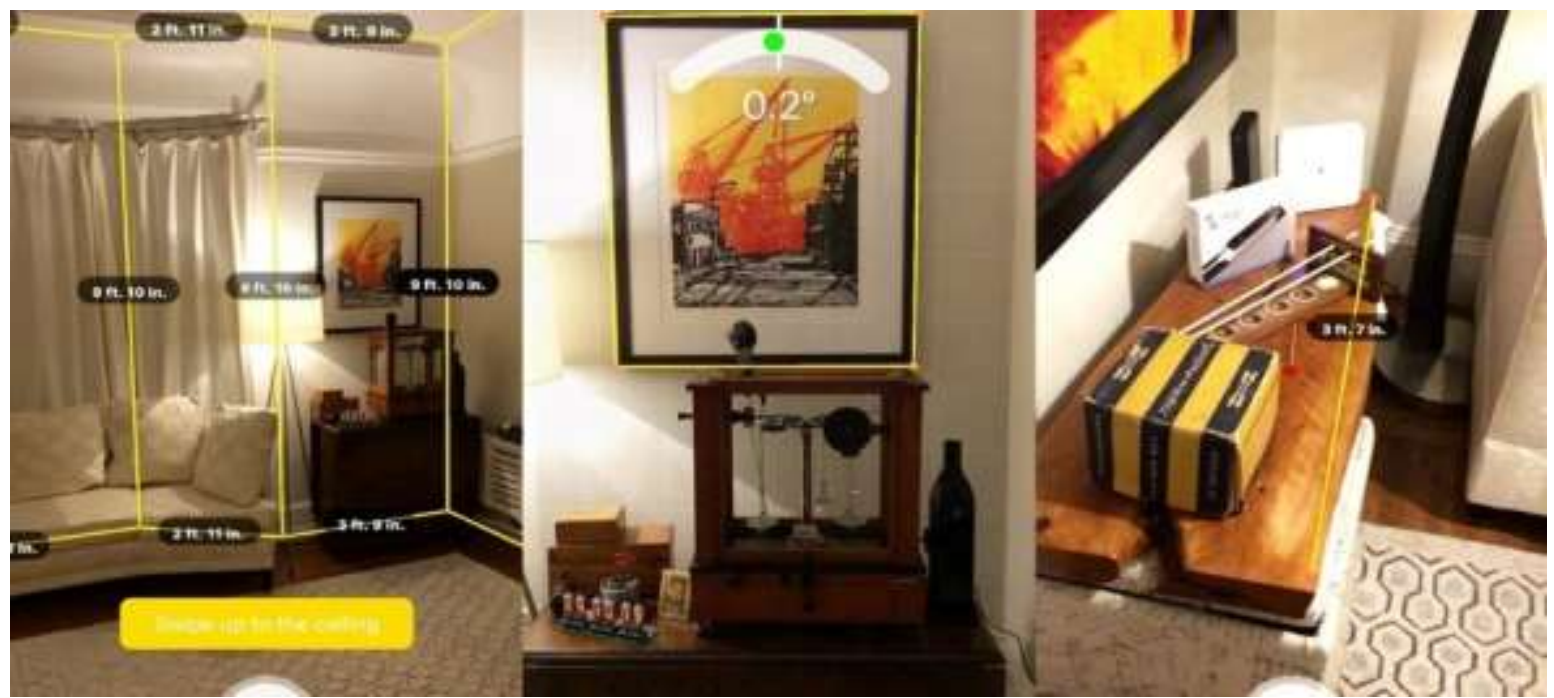

صورة (•) إستخام تطبيق ARKit في عملية التصميم الاخلي، (-0)://mobile-ar.reality.news/news/apple-ar-occipitals-arkit.( Retrieved September,31,2020, 03:50 pm.app-offers-room-scanning-par-with-tango-for-iphones-0180045/ 


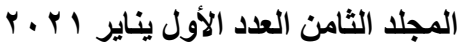

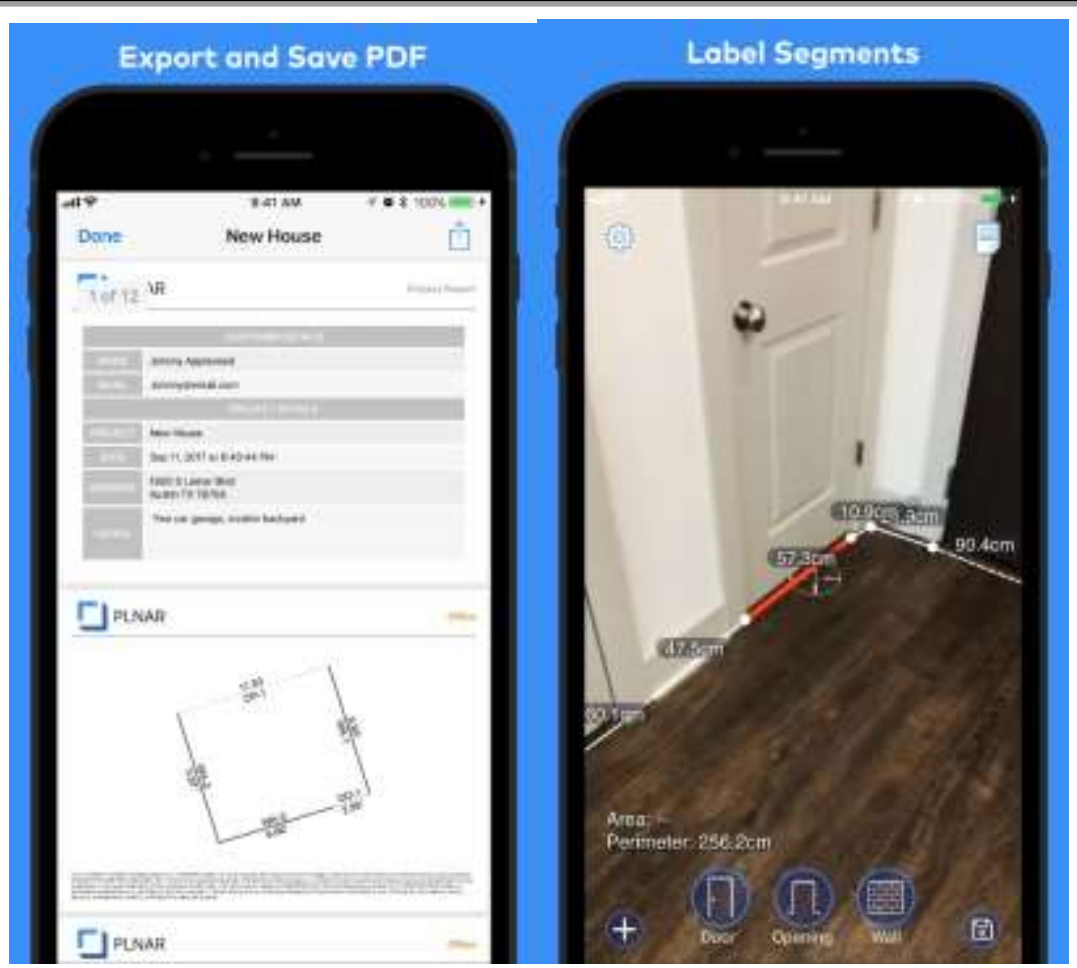

https://mobile-ar.reality.news/news/apple-ar-occipitals-arkit-) صورة (ఛ) إستخام تطبيق ARKit في عملية التصميم الداخلي

.( Retrieved September,31,2020, 03:50 pm.app-offers-room-scanning-par-with-tango-for-iphones-0180045/

r-Y تطييق IKEA place: قدمت ايكيا* مفهوم "المتجر الذكي" لعملائها باستخدام تقنية الواقع المعزز. لقد

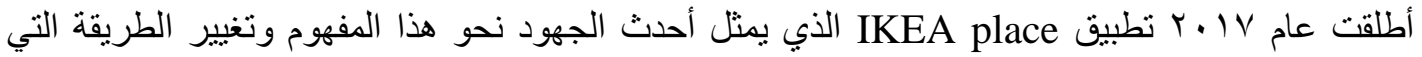

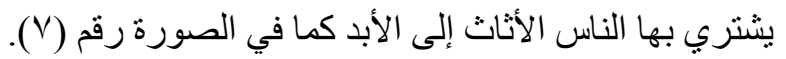
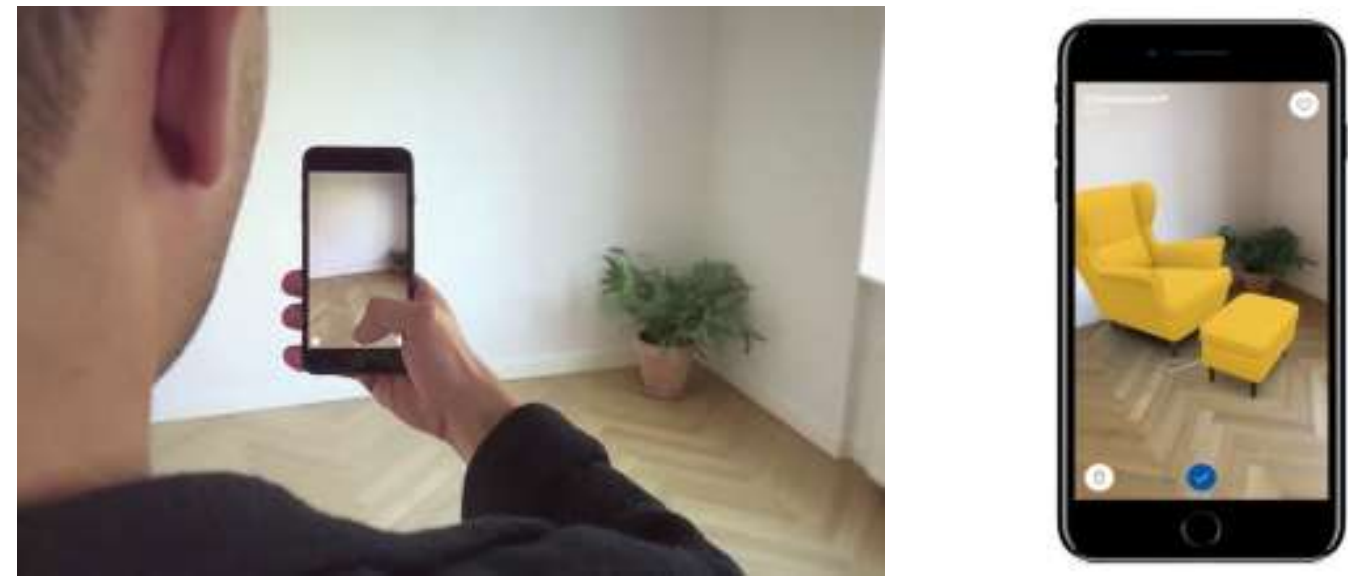

https://www.boredpanda.com/apple-arkit-virtual-(صورة (V) توضح إستخدام تطبيق IKEA place، في التصميم الداخلى

Retrieved furniture-ikea-place-app/?utm source=google\&utm medium=organic\&utm campaign=organic .(August, 31,2020, 05:48 pm

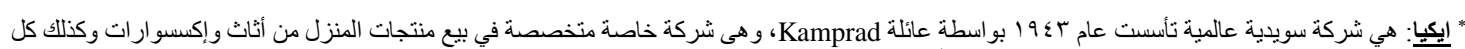

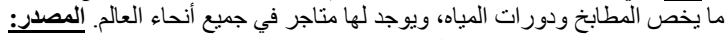
Osama Albarrak, 2011, Case study \& analysis, Ikea. King Saud University College of Business Administration

Journal of Applied Art and Science - International Periodical Scientific Peer Reviewed - Issued By Faculty of Applied Arts - Damietta Univ. Egypt 
r r r تطييق Magicplan IOS: يعد أداه رائدة للتصميم الداخلي و المعماري، ويستخدم في إنشاء المساقط الأفقية

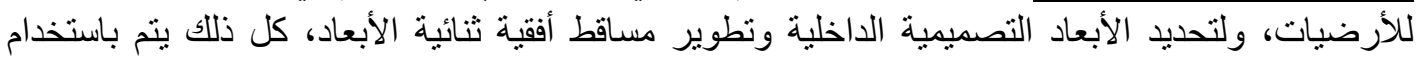

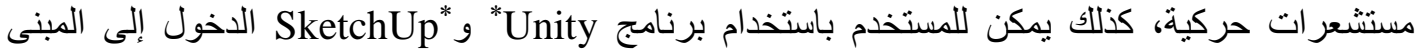

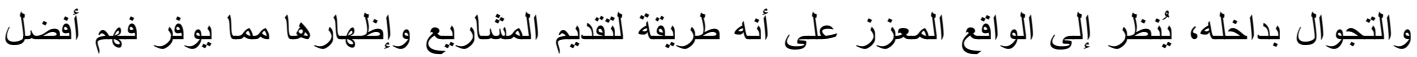

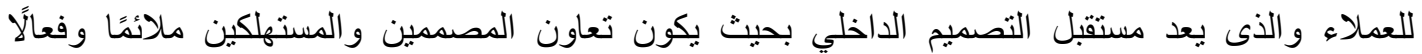

(Gürçınar, E., \& Esen, Ö. C.:2018)

r-s تطيق Home Design 3D

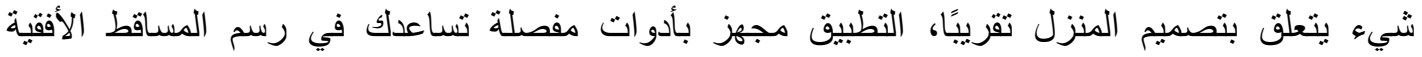

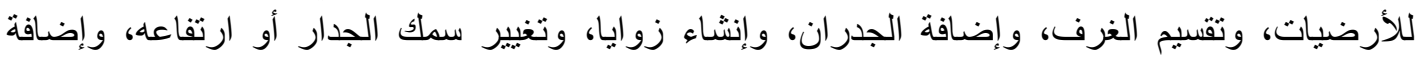

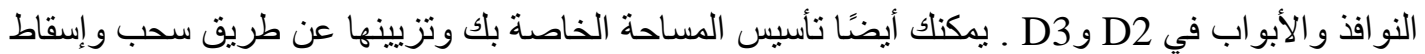

قطع الأثاث و الإكسسوار ات إلى التصميم. كما بالصور رقم (^).

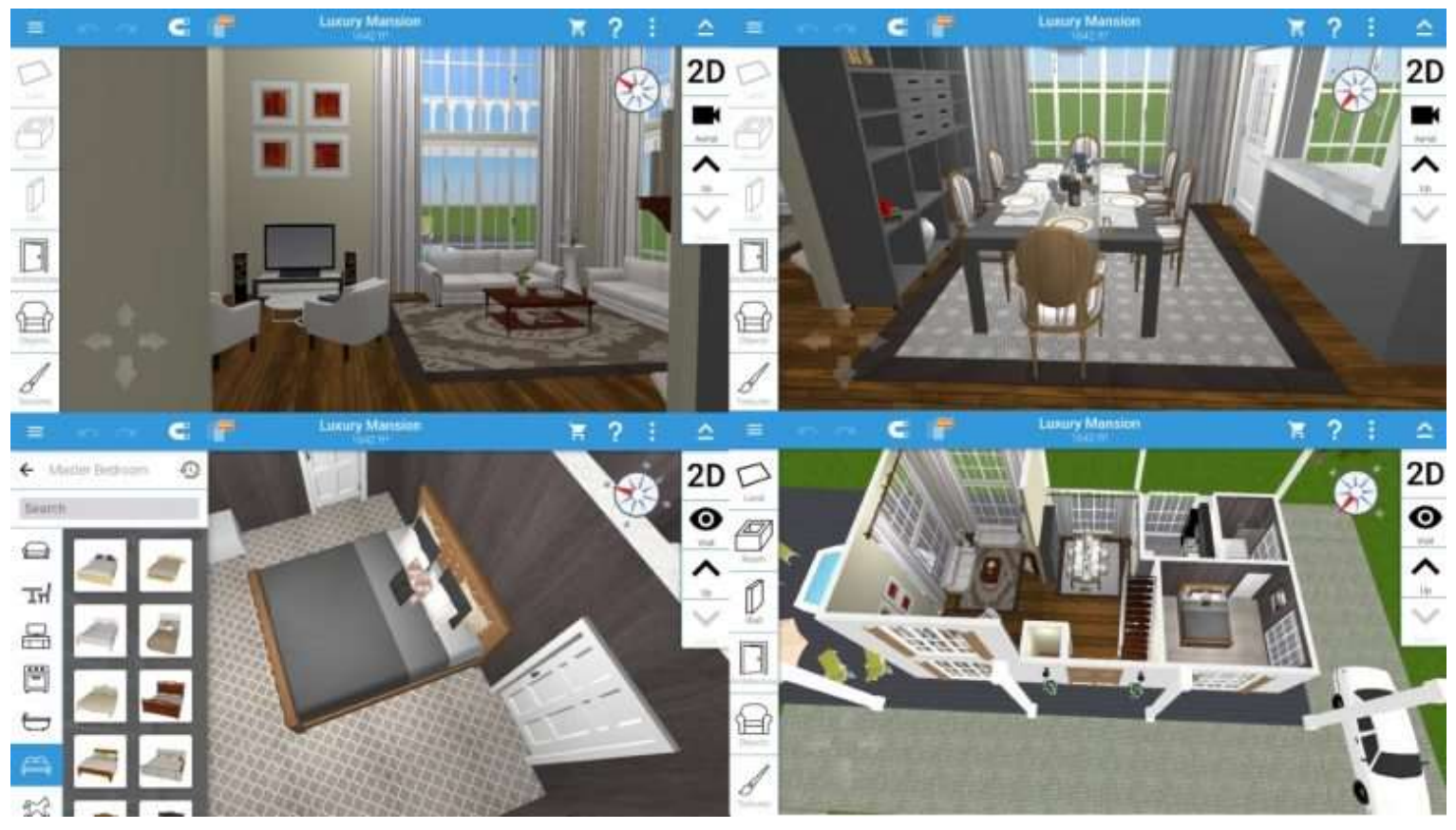

https://www.slashdigit.com/best-.) Home Design 3DD صورة (^) توضح استخدام تطبيق) furniture-design-apps/ Retrieved August,31,2020, at 06:01 pm.)

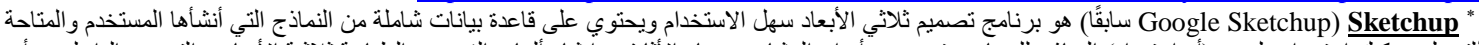

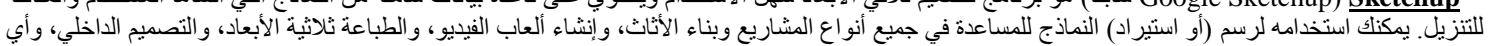
شيء آخر يخطر ببالك. المصدر: Retrieved September,08,2020, at 01:01 PM https://www.howtogeek.com/364232/what-is-sketchup/ 


\section{ع - الواقع المعزز في تصميم المتاحف:

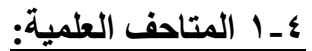

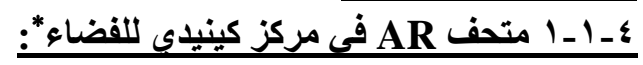

يمكن أن يساعد متحف الو اقع المعزز الناس على فئى فهم الأحداث التاريخية بشكل أفضل. على سبيل المثال، يستخدم

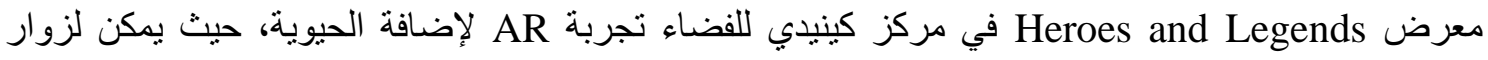

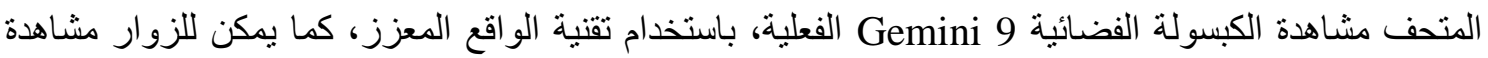

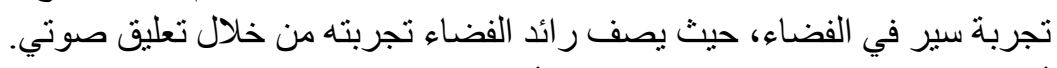

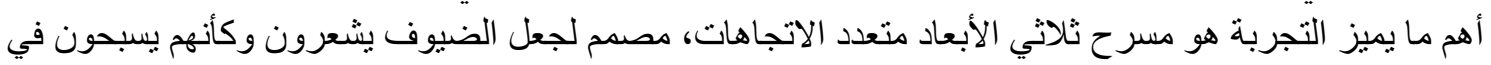

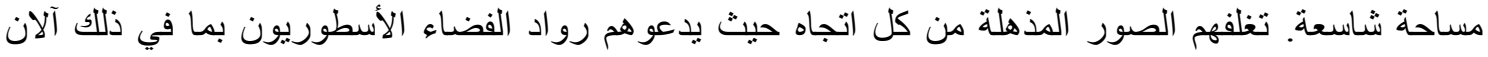

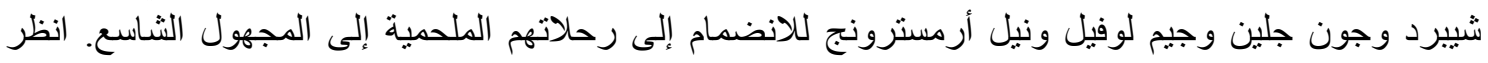

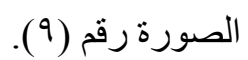

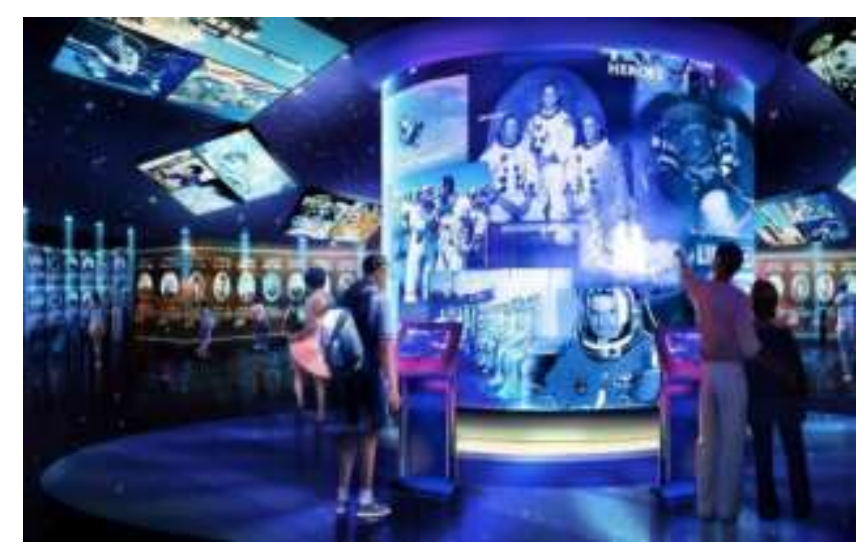

https://spacecoastdaily.com/2015/05/kennedy-) صورة (9) متحف AR في مركز كينيدي للفضاء،

Retrieved space-center-visitor-complex-breaks-ground-for-heroes-and-legends/ .(:32 pm. ') August,23,2020, at 
عندما اكتشفوا الموقع، عثر علماء الآثار على أكثر من

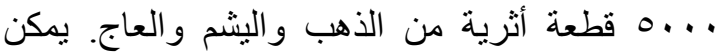

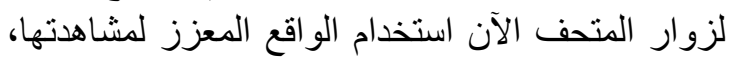

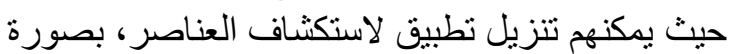
ثلاثية الأبعاد، كما يوفر التطبيق معلومات أكثر تفصيلاً

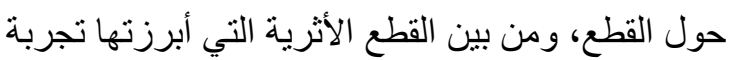

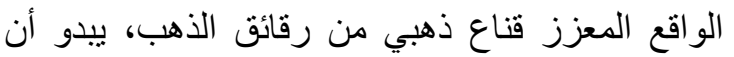

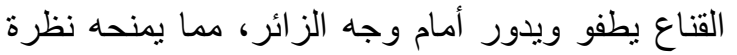
قريبة له بالتفصيل كما بالصورة رقم (• (1).

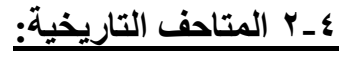

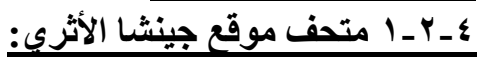

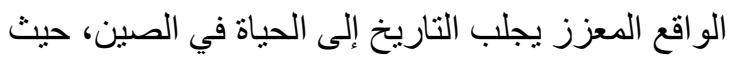

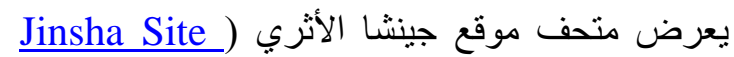
(Museum

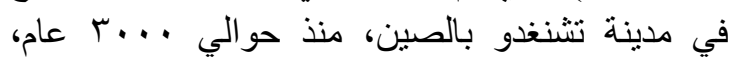

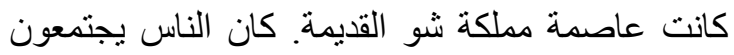

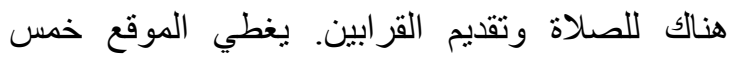

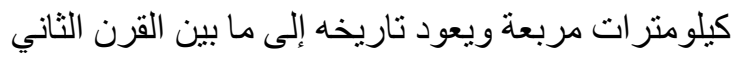
عشر قبل الميلاد و القرن السابع قبل الميلاد.

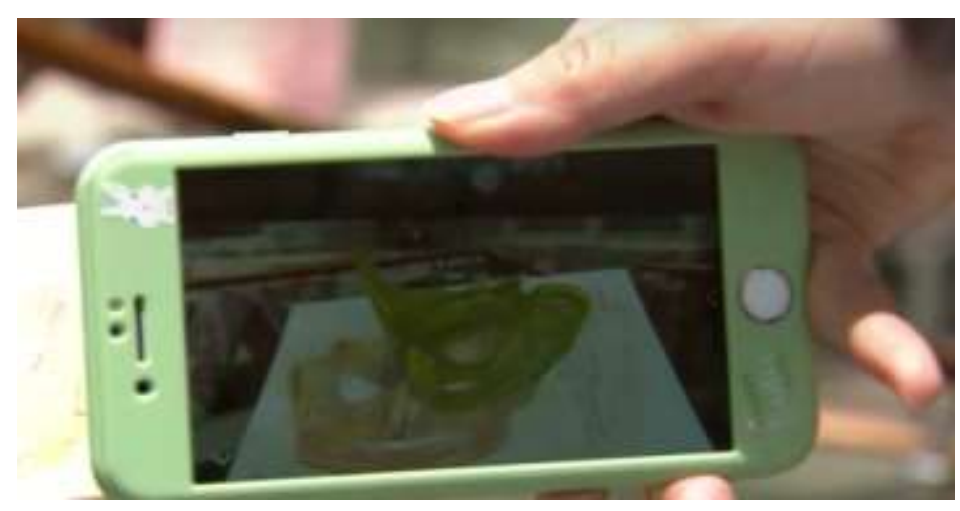

صورة ( • ( ) قناع ذهبي من رقائق الذهب بتقنية الواقع المعزز ،

(https://blooloop.com/features/museum-vr-museum-ar/ Retrieved August,23,2020, at $11: £ 9 \mathrm{pm})$

Hacking the Heist سرقتها وذلك بإستخدام تطبيق

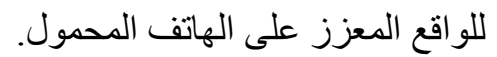
Hacking the Heist

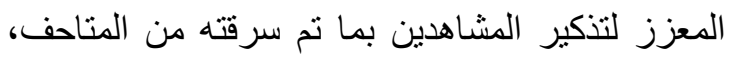

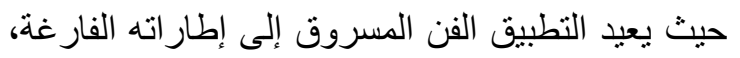

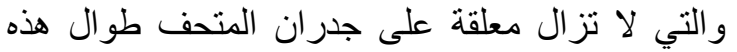

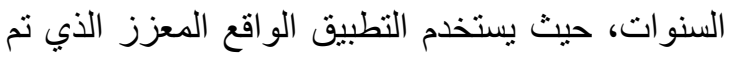
إنشاؤه بواسطة ARKit من Apple. انظر الصورة

رقم (1) - (1).
ع بـ المتاحف القتيةة:

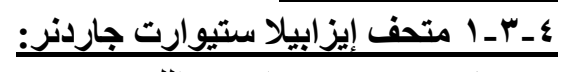

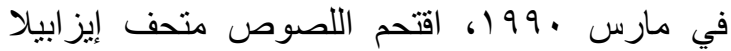

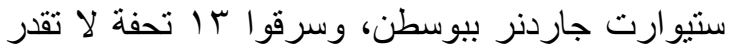

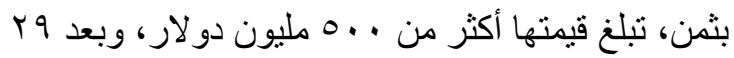

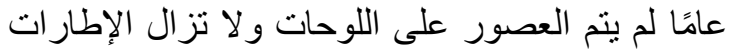

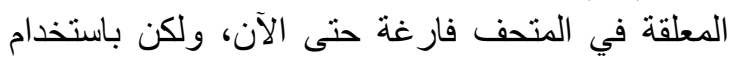

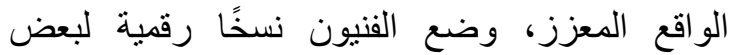
الأعمال الفنية المفقودة في الإطار ات الفرونة الفار غلة، مما يتيح

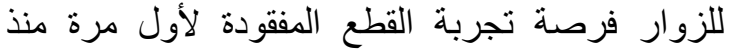




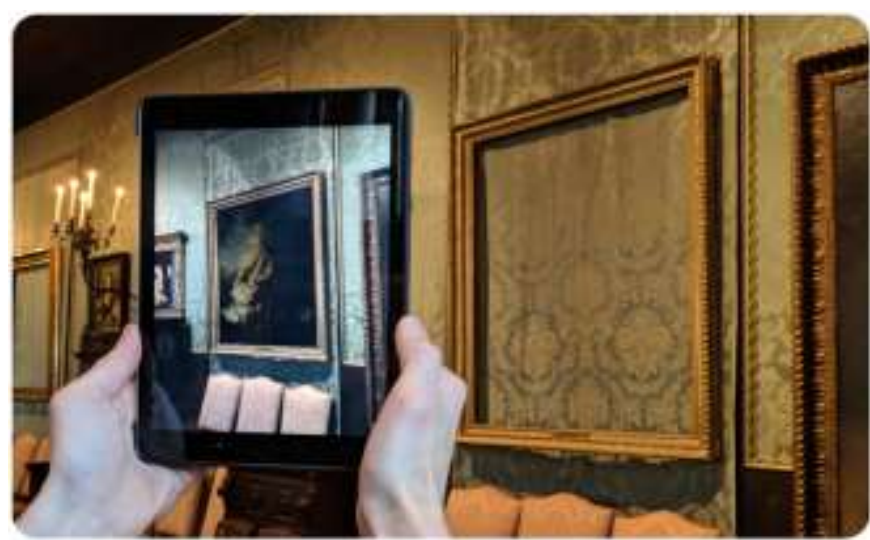

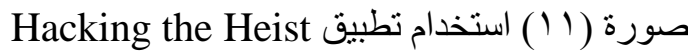

لرؤية اللوحات المفقودة بالمتحف.

(https://www.hackingtheheist.com/ Retrieved August,24,2020, at 12:19 am.)

الثانثة مبانشرة والانغماس على الفور في عالم من هـ - الواقع المعزز والمراكز التجارية:ة

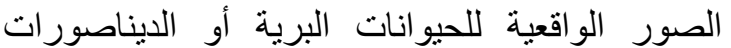

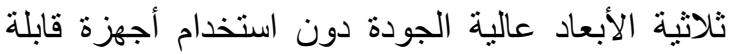
للارتداء باهظة الثمن أو الاضطرار الإدة إلى تنزيل نطبيق،

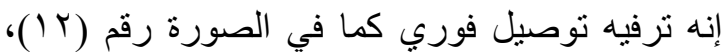

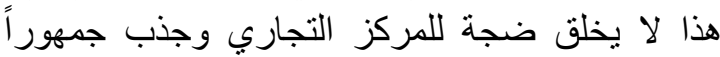
أكبر فحسب، بل يعمل أيضًا على غرس و لاء العملاء.

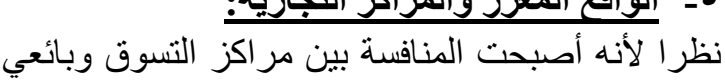

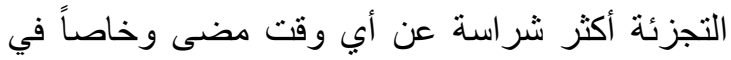

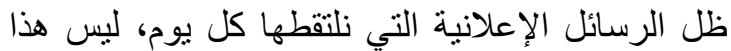

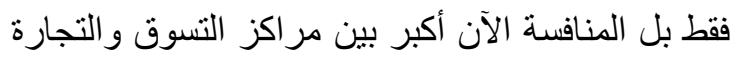

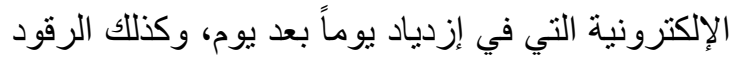

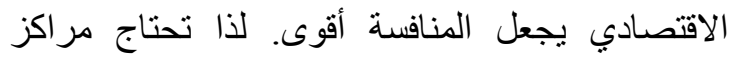
التسوق إلى التركيز على أحداث التنشيط و هنا بأتي دور

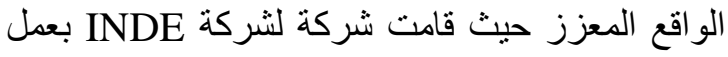
Broadcast AR

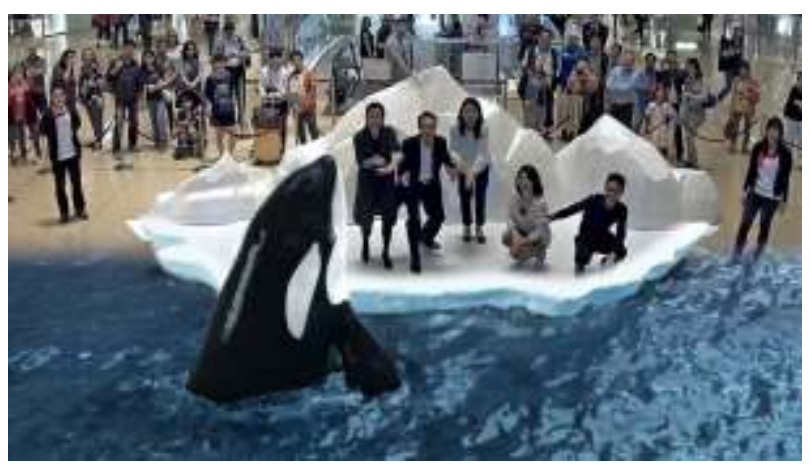

صورة (Y I الو اقع المعزز Broadcast AR.

(https://www.indestry.com/blog/2015/11/12/3-industries-we-target-and-whyaugmented-reality-is-for-everybody Retrieved September,05,2020, at 12:0 • am. )

يمكنك الآن الإحتفال بعيد الميلاد في وسط مركز مارينا هـــ الواقع المعزز في مول مارينا سكوير للتسوق:

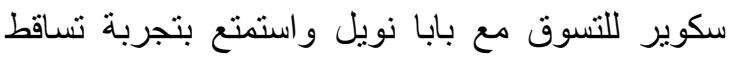


الثلوج بفضل تقنية الواقع المعزز، وأيضاً استمتع مع المخلوقات الثتوية وشاركها مع جميع أصدقائك

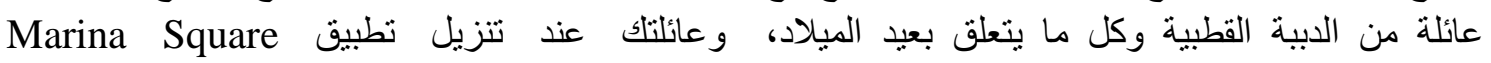

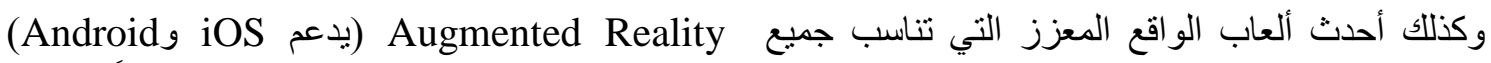

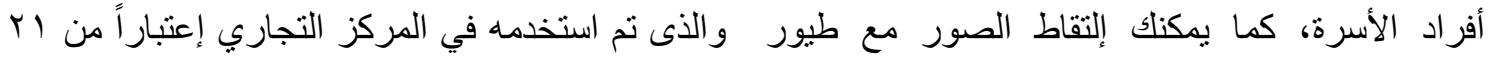

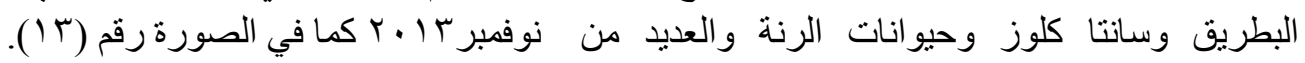
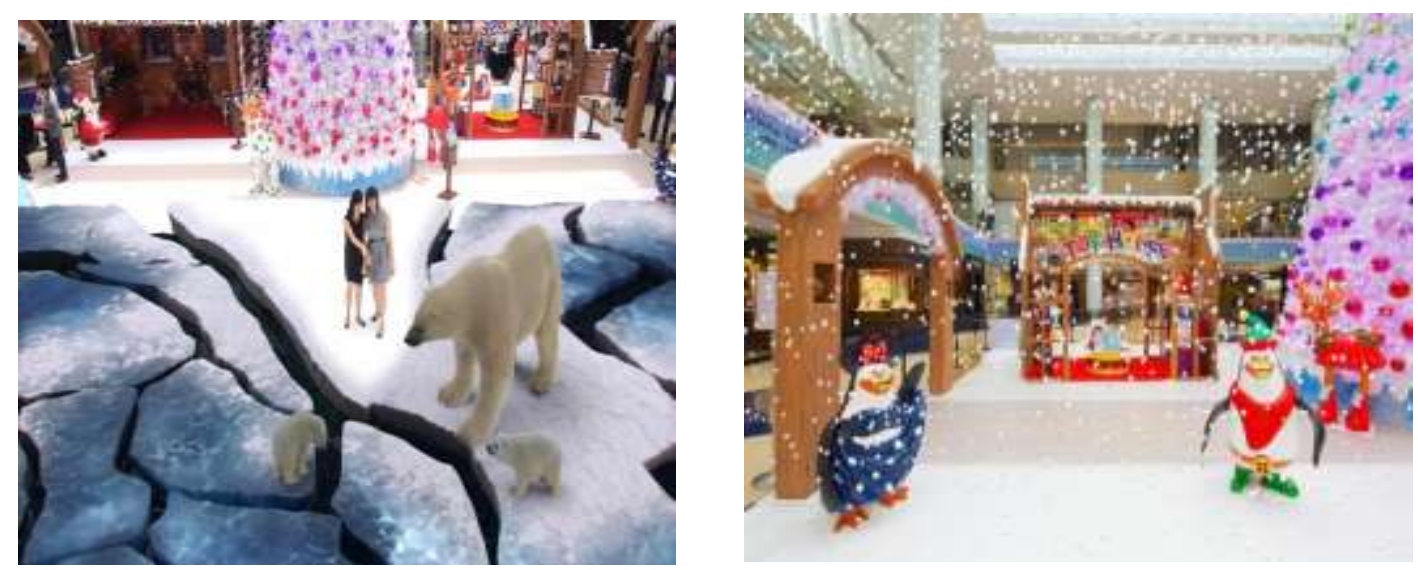

صورة (r ا ) الإحتفال بعيد الميلاد بإستخدام الو اقع المعزز في مول مارينا سكوير للتسوق.

(https://metropolitant.com/2013/12/09/the-largest-augmented-reality-wonderland-atmarina-square-shopping-mall/ Retrieved September,05,2020, at 12:0• am.)

Kreindlin

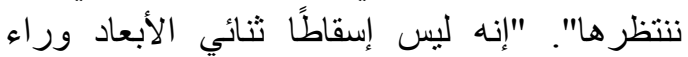
الممثل، ولكنه كائنات ثناثية الأبعاد في الفئل الفضاء

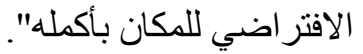

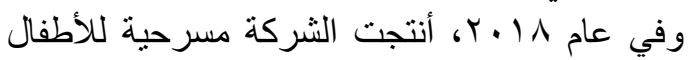
Gulliver تدعم تقنية الواقع المعزز وتم عرضها على مسرح Gesher Theatre، حيث يتم تنبيث الصور التي تم إنشاؤها بواسطة الكمبيوتر فى بيئات

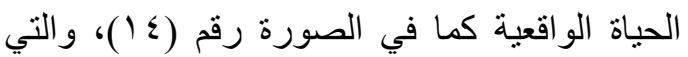

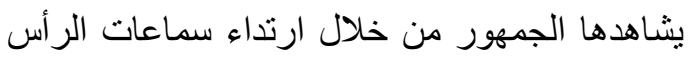

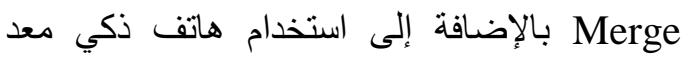

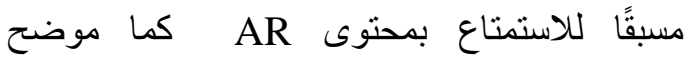

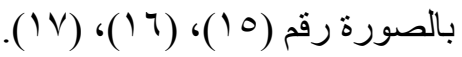

هو هو مالك شركة AR Showa Kreindlin *

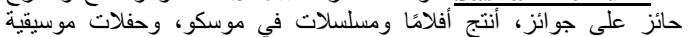
الجمهور يزيد عن .. ألف شخص ومسرحيات شهيرة في مسرح غيشر. Retrieved https://www.linkedin.com/company/arshowpro $5 \mathrm{am} .{ }^{\Upsilon}$ September,08,2020, at 01:
ד- الواقع المعزز والمسرح: طورت شركة ARShow

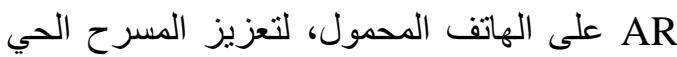

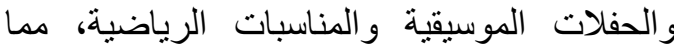

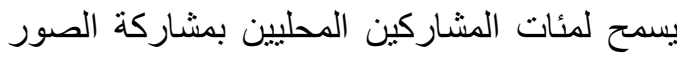

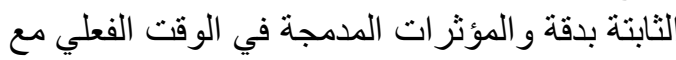
العروض الحية. حيث أعلنت ARShow عن الطناة ونظام تشغيل جديدين يستهدفان دور السينما تحديدًا.

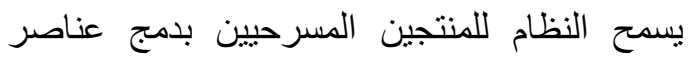
وشخصيات الواقع المعزز الخيالية في العروض لأنسين

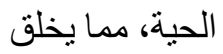
تجارب جماعية محفزة بصريًا تكون عبارة عن النية

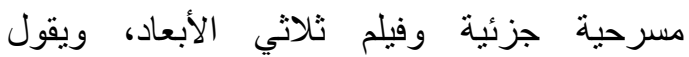

$$
\text { هي شركة إسر ائلية مقر ها تل أبيب، تم نأسيسها بو اسطة :AR Show* }
$$
Sasha Kreindlin http://www.arshowpro.com/arshow-in-forbes-ar-is-the-starRetrieved September,06,2020, at of-a-new-theater-company/ am. $r \cdot: \cdot 1$ 


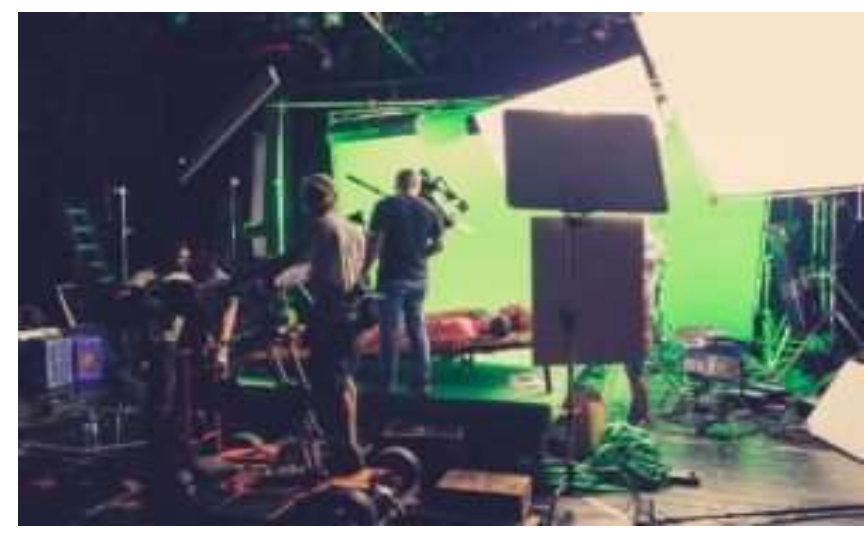

صورة (ع ( ) أثناء التحضير لمسرحية Gulliver على مسرح Gesher Theatre. (http://www.arshowpro.com/they-said-weve-gone-mad-that-augmented-reality-is-forpokemon-go-not-for-theater/ Retrieved September,07,2020, at 10: ${ }^{\top} \mathrm{l}$ am.)

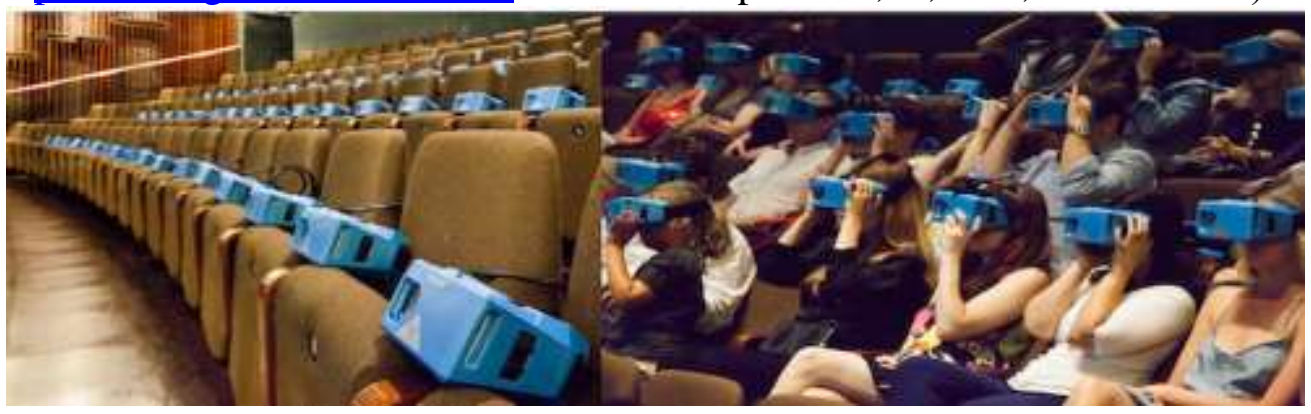

صورة (0 1) يجد كل فرد من الجمهور سماعة رأس Merge soft مزودة بهاتف ذكي قادر على عرض الو اقع المعزز.

(http://www.arshowpro.com/arshow-in-forbes-ar-is-the-star-of-a-new-theater-company/ Retrieved September,06,2020, at $1 \cdot: r \cdot$ AM )

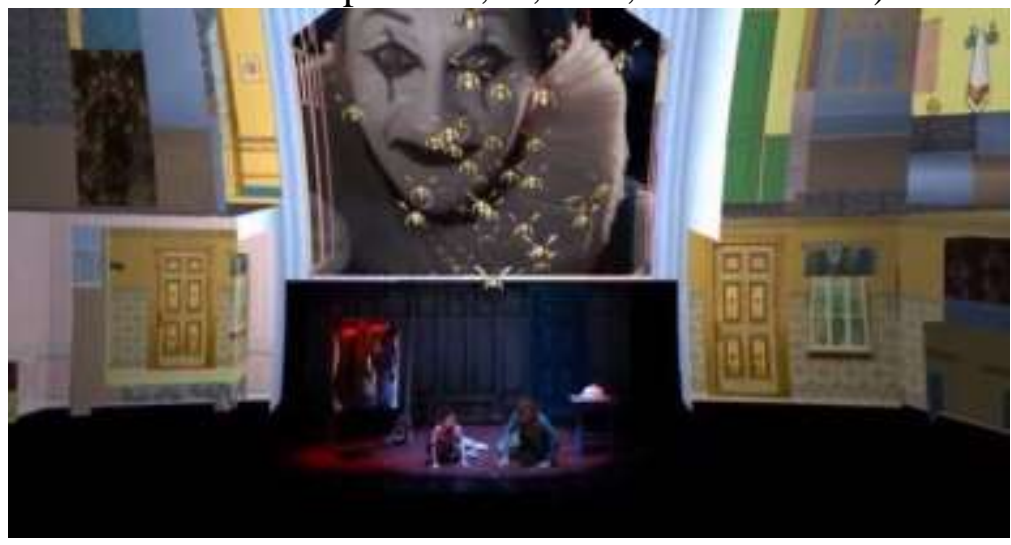

صورة (7 1 ) الو اقع المعزز لمسرحية Gulliver على مسرح Gesher Theatre

(https://www.forbes.com/sites/charliefink/2018/11/05/ar-is-the-star-of-new-theatercompany/\#65e9c6051d8e Retrieved September,06,2020, at 11:0• PM.) 


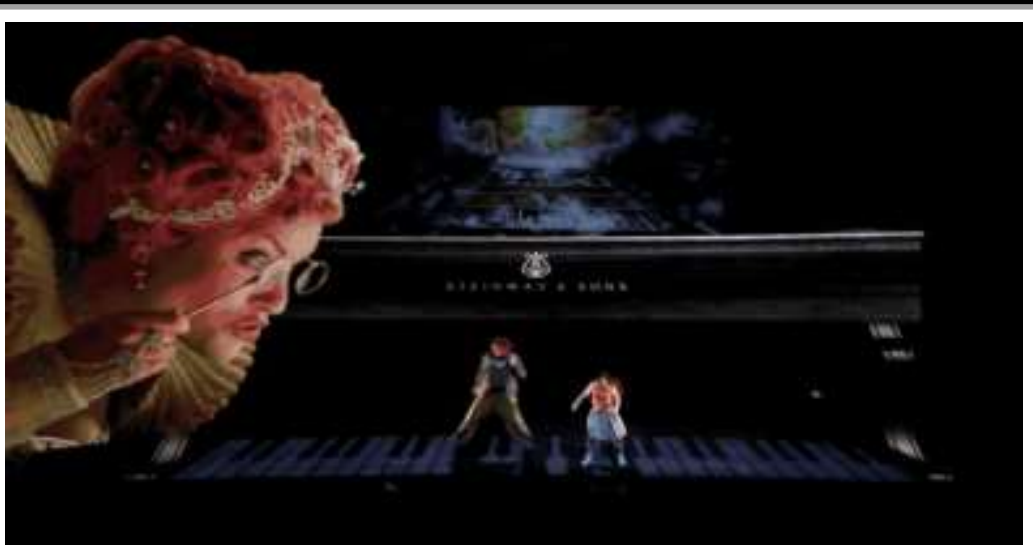

صورة (V) الو اقع المعزز لمسرحية Gulliver على مسرح Gesher Theatre.

(http://ciopride.com/2018/06/15/meet-the-ar-startup-that-wants-to-give-live-theater-anaugmented-makeover/ Retrieved September,06,2020, at 11:01 PM.)

\section{V- - الواقع المعزز و الحفلات الترفيهيه:}

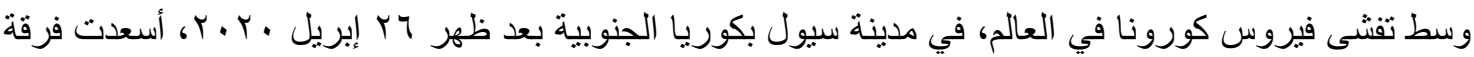

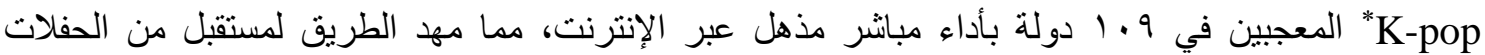

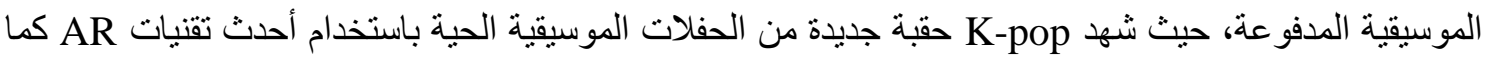

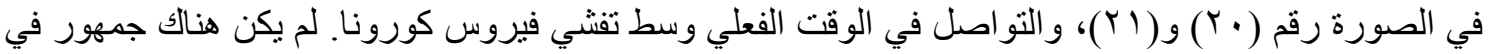

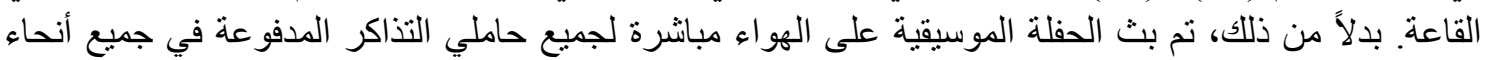

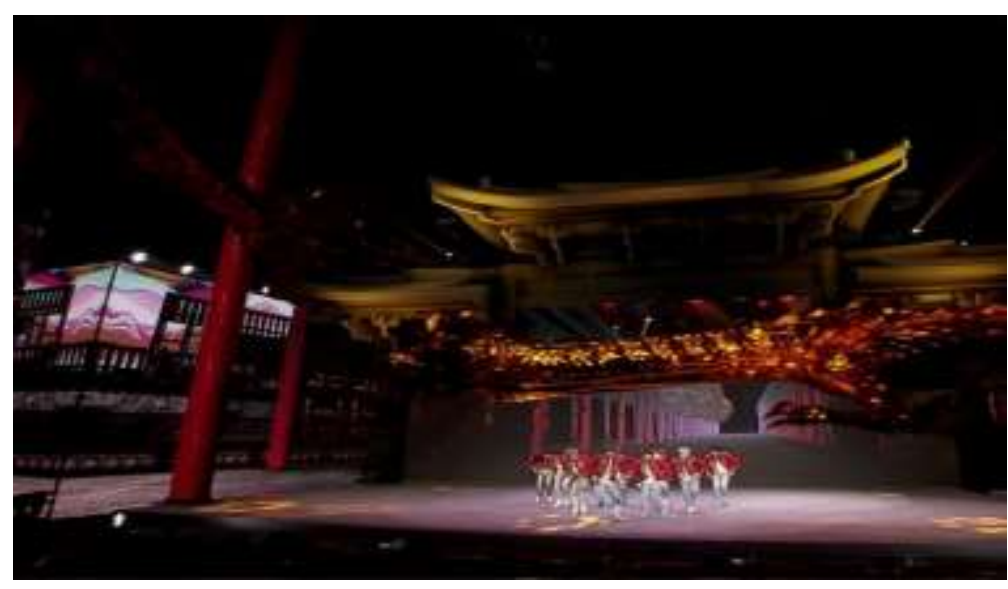

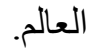

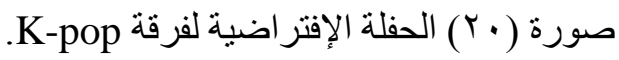

(https://davidbluesky.online/k-pop-groups-are-providing-immersive-virtual-concerts/ Retrieved September,07,2020, at 09:10 PM.)

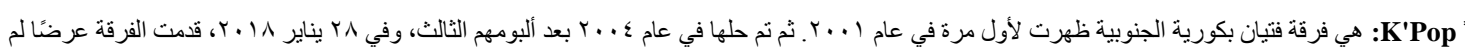

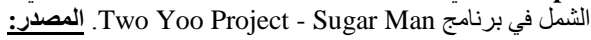

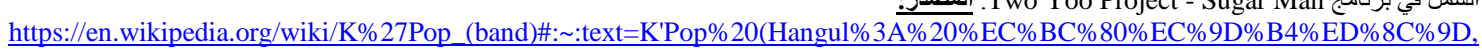
. Retrieved September,08,2020, at 01:34 AM.after\%20their\%20third\%20album\%2C\%20Memories

Journal of Applied Art and Science - International Periodical Scientific Peer Reviewed - Issued By Faculty of Applied Arts - Damietta Univ. Egypt (ISSN 2537-1061) (Print) 


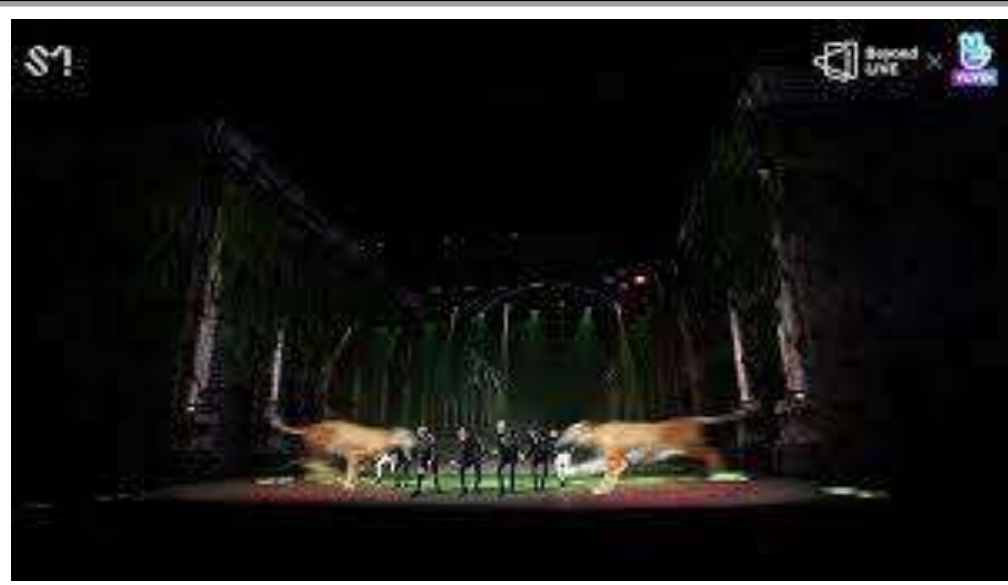

$$
\begin{aligned}
& \text { صورة ( Y (Y) الحفلة الإفتر اضية لفرقة K-pop على خشبة المسر ح أمام جمهور افتر اضي، }
\end{aligned}
$$

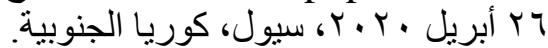

(http://www.sisanews.kr/news/articleView.html?idxno=48698 Retrieved

September,0^,2020, at 02: or AM.)

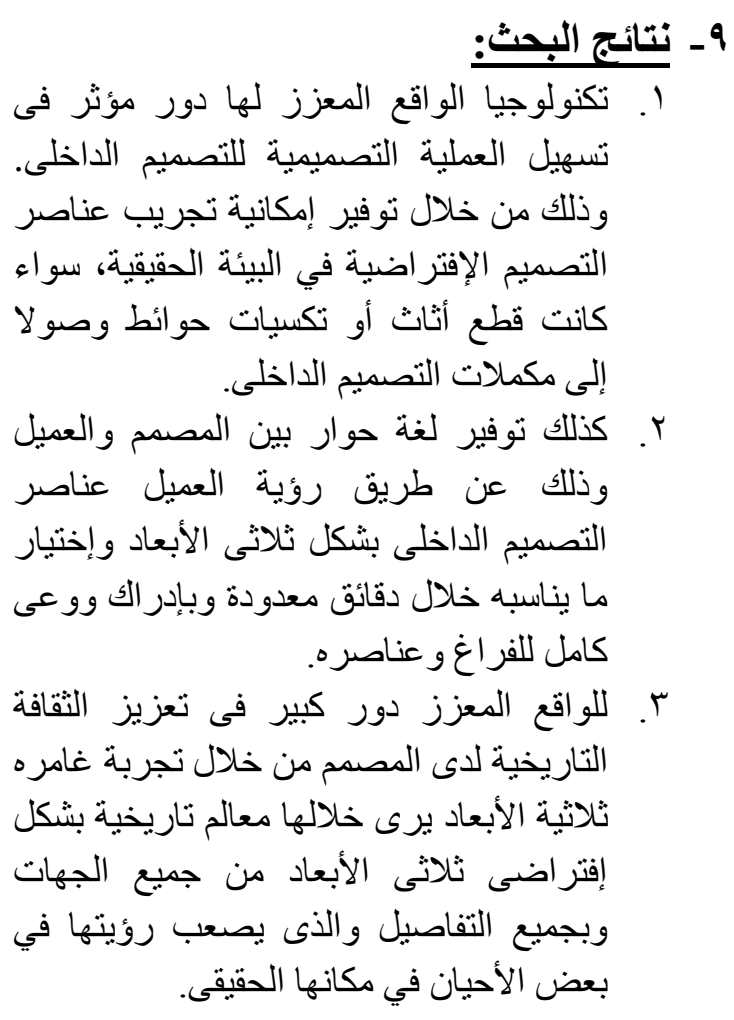

1. Alkhamisi, A. O., Arabia, S., \& Monowar, M. M. (2013). Rise of augmented reality: Current and future application areas. International journal

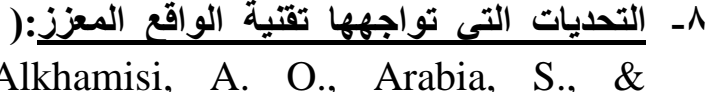

(Monowar, M. M.: 2013

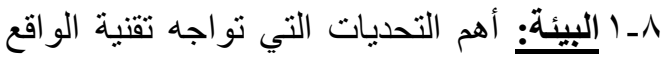

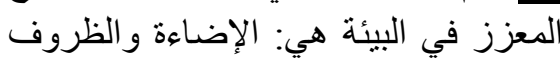

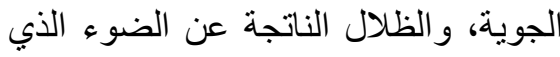

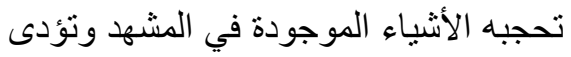

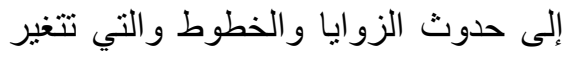

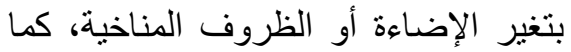

يؤدي تنوع الألوان في البيئة إلى مشكلة في الإن الني

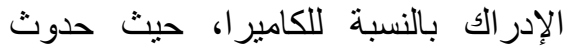

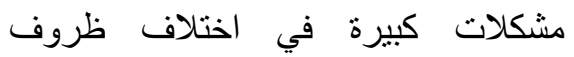

الإضـاءة، كما تؤثر الأسطح ذات التئلات التباينات

العالية في اللون على على العكاس الصنط ذات الصور

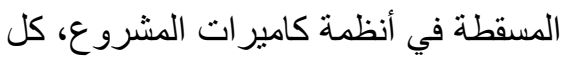

ذللك يؤثر على الصورة الإفتراضية الناتجة

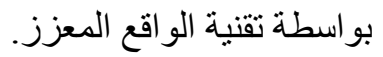

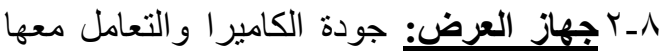

في ظروف الإضـاءة السيئة، يؤدى إلى اللى الى الى

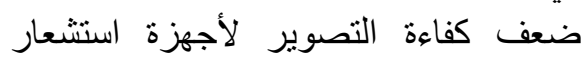

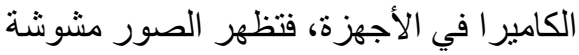

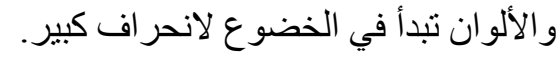

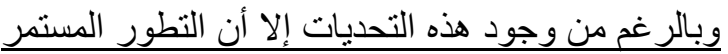

و السريع في التكنولوجيا يعمل على تخطي كل التحديات إلاتئ التطور المبني. 
environment. International Journal of Computer Applications, 5(5), 16-21.

9. Sandu, M., \& Scarlat, I. S. (2018) Augmented Reality Uses in Interior Design. Informatica Economica, 22(3).

10. Siltanen, S. (2015). Developing augmented reality solutions through user involvement, PHD, Aalto University School of Science.

11. Wang, J., Wang, X., Shou, W., Xu, B., Wang, J., \& Xu, B. (2014). Integrating BIM and augmented reality for interactive architectural visualization.

https://doi.org/10.1108/CI- 03-20140019.

12. Wang, X. (2009). Augmented reality in architecture and design: potentials and challenges for application. International Journal of Architectural Computing, 7(2), 309326.

13. http://ciopride.com/2018/06/15/meetthe-ar-startup-that-wants-to-give-livetheater-an-augmented-makeover/ Retrieved September,06,2020, at 10:r5 am.

14. http://www.arshowpro.com/arshow-inforbes-ar-is-the-star-of-a-new-theatercompany/ Retrieved September,06,2020, at 10:30 am.

15. http://www.arshowpro.com/they-saidweve-gone-mad-that-augmentedreality-is-for-pokemon-go-not-fortheater/ Retrieved September,07,2020, at 10:31 am.

16. http://www.sisanews.kr/news/articleVi ew.html?idxno=48698 Retrieved September,08,2020, at 02:53 AM.

17. https://abcnews.go.com/International/p op-boy-band-superm-thrills-fans-
of internet
and
distributed
systems, 1(04), 25.

2. B.Y.Jani, Pratiksha Dahale, Ankita Nagane, Bhavika Sathe, Nilam Wadghule, (2015), Interior Design in Augmented Reality Environment, International Journal of Advanced Research in Computer and Communication Engineering, Vol. 4, Issue 3

3. Chang, Y. S., Hu, K. J., Chiang, C. W., \& Lugmayr, A. (2020). Applying Mobile Augmented Reality (AR) to Teach Interior Design Students in Layout Plans: Evaluation of Learning Effectiveness Based on the ARCS Model of Learning Motivation Theory. Sensors, 20(1), 105.

4. David Mccartney,(2014), Introduction To Autocad Made Easy For Beginners.

5. Gürçınar, E., \& Esen, Ö. C. (2018). The Application of Augmented Reality in Interior Design Education. DS 91: Proceedings of Nord Design 2018, Linköping, Sweden, 14th-17th August 2018.

6. Lim, D. H., Han, S. J., Oh, J., \& Jang, C. S. (2019). Application of virtual and augmented reality for training and mentoring of higher education instructors. In Handbook of research on virtual training and mentoring of online instructors (pp. 325-344). IGI Global.

7. Osama Albarrak, (2011), Case study $\&$ analysis, Ikea. King Saud University College of Business Administration

8. Phan, V. T., \& Choo, S. Y. (2010). Interior design in augmented reality 
theatre/ Retrieved September,06,2020, at 10:31 am.

26. https://spacecoastdaily.com/2015/05/k ennedy-space-center-visitor-complexbreaks-ground-for-heroes-and-legends/ Retrieved August,23,2020, at 11:32 pm.

27. https://web.ics.purdue.edu/ fwinkler/A D41700_F13/AD41700_Unity3D_wor kshop01_F13.pdf

28. https://www.boredpanda.com/applearkit-virtual-furniture-ikea-placeapp/?utm_source=google\&utm_mediu $\underline{\mathrm{m}=\text { organic\&utm_campaign }=\text { organic }}$ Retrieved August,31,2020, 05:48 pm.

29. https://www.eenewseurope.com/news/ dynamic-led-wall-replaces-greenscreens-ar

Retrieved September,05,2020, at 10:0 · PM.

30. https://www.fastcompany.com/405502 00/this-ar-app-takes-you-behind-thescenes-of-the-worlds-most-famous$\underline{\text { art- }}$ heist\#: :text=Hacking\%20the\%20Heis t\%20is\%20a,AR\%20built\%20with\%2 OApple's\%20ARKit. Retrieved August,24,2020, at 12:17 am.

31. https://www.forbes.com/sites/charliefi nk/2018/11/05/ar-is-the-star-of-newtheater-company/\#65e9c6051d8e Retrieved September,06,2020, at 11:0 • PM.

32. https://www.hackingtheheist.com/

Retrieved August,24,2020, at 12:19 am.

33. https://www.howtogeek.com/364232/ what-is-sketchup/ Retrieved September,08,2020, at 01:01 PM

34. https://www.indestry.com/blog/2015/1 $\underline{1 / 12 / 3 \text {-industries-we-target-and-why- }}$ augmented-reality-is-for-everybody performsconcert/story? $\mathrm{id}=70362270$

Retrieved September,07,2020, at 09:05 PM.

18. https://ar.wikipedia.org/wiki/\%D9\%85 \%D8\%B1\%D9\%83\%D8\%B2 \%D9\% 83\%D9\%8A\%D9\%86\%D9\%8A\%D8 \%AF\%D9\%8A \%D9\%84\%D9\%84\% D9\%81\%D8\%B6\%D8\%A7\%D8\%A1 Retrieved September,08,2020, at 01:01 $\mathrm{AM}$

19. https://blooloop.com/features/museumvr-museum-ar/ Retrieved August,23,2020, at 11:14 pm.

20. https://davidbluesky.online/k-popgroups-are-providing-immersivevirtual-concerts/ Retrieved September,07,2020, at 09:10 PM.

21. https://en.wikipedia.org/wiki/K\%27Po p_(band)\#: :text=K'Pop\%20(Hangul\% 3A\%20\%ЕC\%BC\%80\%ЕC\%9D\%B4 $\% \mathrm{ED} \% 8 \mathrm{C} \% 9 \mathrm{D}$, after\%20their\%20third $\% 20$ album $\% 2 \mathrm{C} \% 20 \mathrm{Memories}$.

Retrieved September,08,2020, at 01:34 AM.

22. https://en.wikipedia.org/wiki/Temple of_Hera,_Olympia Retrieved August,31,2020, 03:14 pm.

23. https://metropolitant.com/2013/12/09/t he-largest-augmented-realitywonderland-at-marina-squareshopping-mall/ Retrieved September,05,2020, at 12:00 am.

24. https://mobilear.reality.news/news/apple-aroccipitals-arkit-app-offers-roomscanning-par-with-tango-for-iphones0180045/ Retrieved September,31,2020, 03:50 pm.

25. https://rewind.co/news/howtechnology-is-augmenting-traditional- 
المجلد الثامن العدد الأول يناير ا Y r r

Reality-Environment-Phan

Choo/6f335173a4608f4e3c38837f8d0c

7ed7d0b52208

August,31,2020, 03:50 pm.

Retrieved

37. https://www.slashdigit.com/best-

furniture-design-apps/

August,31,2020, at 06:01 pm.
Retrieved September,05,2020, at 12:0 • am.

35. https://www.linkedin.com/company/ar showpro

Retrieved September,08,2020, at 01:r5 am.

Retrieved 36. https://www.semanticscholar.org/paper /Interior-Design-in-Augmented- 


\section{The impact of augmented reality technology on the interior design}

\section{Research Summary:}

The 21 st century has been characterized by a high rate of development, especially in the field of technology, and due to the digital economy's effort to support artificial intelligence technology, which has already started accompanying us in daily work and entertainment, which gives an opportunity to achieve innovations, so the designer is looking for new technologies to be able to develop his abilities. By including digital media in the design process, this has led to discover new fields of design as computer aid design.

Hence, the problem of research appears due to the difference in perception levels of people. Also, Traditional methods of visualization are usually show one point of view, so reality techniques as augmented reality were used to help people to understand the relationships between 3D shapes. The importance of this research lies in introducing new designs for interior design field that matches the era of development. The research is based on the descriptive analytical approach. The research aims to shed light on augmented reality technology and its applications in interior design, theater and cinema decoration.

As a results, augmented reality has an influential role in facilitating the design process, and also helps to provide understanding between the designer and the client, it also has a great role in enhancing the designer's historical culture, and plays an important role in furniture marketing, Therefore, researchers are recommended to expand the exploitation of technology that serves interior design and furniture such as augmented reality technology. 